\title{
QUANTITATIVE REMOTE SENSING ANALYSIS OF THERMAL ENVIRONMENT CHANGES IN THE MAIN URBAN AREA OF Guilin BASED ON GEE
}

\author{
Peiqing Lou, Bolin Fu*, Xingchen Lin, Tingyuan Tang, Lu Bi \\ College of Geomatics and Geoinformation, Guilin University of Technology, Guilin 541004
}

China-gislou@126.com (L.P.); fb12012@126.com (F.B.); linxingchen2019@126.com (L.X.); Tangty@126.com (T.T.);

bilu1017@126.com (B.L.)

\section{Commission VI, WG VI/4}

KEY WORDS: Thermal Environment, GEE, Mono-window Algorithm, Random Forest Algorithm, Landsat 8, Dynamic Analysis

\begin{abstract}
:
The dynamic change of urban thermal environment caused by the change of land use type has become one of the important problems of urban ecological environment protection. In Guilin city as research area, based on the Google Earth Engine (GEE), the random forest algorithm was used to classify the land use classification of Landsat remote sensing images in 2010, 2014 and 2018, and the mono-window algorithm was used to calculate the surface temperature. The surface vegetation was solved according to the NDVI pixel binary model. Coverage, and finally dynamic statistics and comparative analysis of land use, vegetation cover and surface temperature. The main results as follows. (1) From 2010 to 2018, the average temperature in the main urban area of Guilin is on the rise (increased by $1.29^{\circ} \mathrm{C}$ ), and the temperature zones in each class are converted from low temperature zone, lower temperature zone and medium temperature zone to higher temperature zone and high temperature zone. (2) Lower temperature zone and the low temperature zone is mainly distributed in vegetation and water body coverage areas, while the medium temperature zone, higher temperature zone and the high temperature zone are mainly distributed in construction land and unused land cover area. (3) High vegetation cover area in 2014-2018 (reduced by 31.34\%) The main reason for the sharp decline is the substantial increase in the area of construction land (expansion 30.19\%). (4) GEE-based random forest algorithm Land use classification had higher classification accuracy (more than $80 \%$ in all three periods). The results can provide scientific basis for improving urban thermal environment and scientific reference for the development strategy of Guilin city.
\end{abstract}

\section{INTRODUCTION}

The urban thermal environment is an important parameter of the urban ecological environment (Xu, 2015; Fu et al, 2018; Meng et al, 2018). Satellite remote sensing can provide earth observation data in a short period of time, which is an important means to obtain surface temperature (Hou et al, 2018). The use of satellite remote sensing for dynamic analysis of urban thermal environment and its influencing factors is of great significance.

The current land use change caused by land use has brought about a series of ecological and environmental problems, such as the large reduction of vegetation coverage area (Zhang et al, 2018), the large number of rivers and lakes disappeared (Maimaitijiang et al, 2018), and the heat island effect continued to increase (Xie et al, 2017). In response to these problems, domestic and foreign scholars have carried out research on the impact of land use change on urban thermal environment

\footnotetext{
* Corresponding author
} 
changes in recent years. MODIS and AVHRR surface temperature products are mostly used for surface temperature inversion, but their spatial resolution is low, which is only suitable for large-area research (Hou et al, 2010; Weng et al, 2015). Launched in 2013, the Landsat 8 satellite is equipped with a thermal infrared sensor (TIRS) that provides a large amount of high spatial resolution data, making it ideal for urban-scale surface temperature inversion studies. Based on the Landsat data, Jimenez-Munoz et al. (2014) compares the inversion accuracy of the single-window algorithm with the windowed algorithm. It is found that the accuracy of the window algorithm is slightly higher than that of the single-channel algorithm with the increase of atmospheric water vapor content. Yu et al. (2014) compared the radiation transfer equation method, the window algorithm and the single-channel algorithm. The results show that the radiation transfer equation method has the highest accuracy. The US Geological Survey (USGS) pointed out that there is calibration instability in the TIRS 11 band, and it is not recommended to use the window algorithm to invert the surface temperature (Hu et al, 2017). Although Wang (2010) and Liang (2012) have studied the surface temperature inversion in Guilin, they have not analyzed the impact mechanism between land use change and vegetation coverage and surface temperature, and a set of Research techniques for real-time rapid surface temperature inversion.

The Google Earth Engine (GEE) is an open source intelligent cloud platform for satellite imagery and other spatial data solutions (Xiu et al, 2019). GEE provides multi-space scale, multi-source remote sensing data such as Landsat TM/OLI, Sentinel-1/2, MODIS and DMSP/OLS lighting data on a global scale, with a capacity of PB and increasing every day. GEE breaks the traditional remote sensing software download data, preprocessing, information extraction, and analysis applications to obtain thematic information (Tian et al, 2019). Great convenience for remote sensing workers.

Based on GEE's multi-temporal Landsat remote sensing data, this paper dynamically analyzes the thermal environment in the main urban area of Guilin, and explores the relationship between land use types, vegetation coverage and surface temperature, etc., as an optimization of urban ecological environment. Important reference. The research results provide technical support for urbanization construction and green space construction in Guilin, and provide reasonable suggestions for mitigating the urban heat island effect and building a livable city.

\section{DATA SOURCE AND PROCESSING}

\subsection{Study area}

Guilin City is located in the northeastern part of Guangxi Zhuang Autonomous Region, with a longitude of $109^{\circ} 36^{\prime} 50^{\prime \prime} \sim$ $111^{\circ} 29^{\prime} 30^{\prime \prime}$ east, $24^{\circ} 15^{\prime} 23^{\prime \prime} \sim 26^{\circ} 23^{\prime} 30^{\prime \prime}$ north latitude, and 154 meters above sea level in the city center. The karst (karst) landform is a world-famous tourist city, the gateway city of the ASEAN Free Trade Area, and an important connecting point of the "Belt and Road". This paper takes the main urban area of Guilin (Xiufeng District, Diecai District, Xiangshan District, Qixing District) as the research area, with a total area of about 286 square kilometers (Figure.1). The main urban area is located at low latitude and belongs to subtropical monsoon climate. The annual average temperature is nearly $19.1^{\circ} \mathrm{C}$. The hottest temperatures in July and August are about $28{ }^{\circ} \mathrm{C}$. The coldest temperatures in January and February are about $9{ }^{\circ} \mathrm{C}$.

\subsection{Data source}

(1) Landsat imagery: 2010 Landsat TM imagery, Landsat OLI imagery in 2014 and 2018 are provided by the GEE Platform., based on GEE API programming, screening Landsat imagery from November 1st to December 1st of that year with less than $8 \%$ cloud cover The final selected Landsat imaging imaging time was November 14, 2010, November 14, 2014 and November 17, 2018.

(2) Other data: SRTM DEM data space resolution is $30 \mathrm{~m}$, provided by NASA (National Aeronautics and Space Administration), can be directly called in GEE. MODIS L1B Calibrated Reflectance products can also be directly called in GEE, which is mainly used to estimate the atmospheric water vapor content in the study area. The imaging time is November 14, 2010, November 14, 2014 and November 17, 2018, respectively. Consistent with Landsat images. The training sample data and accuracy verification data are selected from the visual interpretation of Google Earth Pro; the surface temperature data is derived from the daily meteorological data of the national meteorological site provided by the China Meteorological Data Network (http://data.cma.cn/). The data set, the acquisition time is consistent with Landsat, and is mainly used for the surface temperature inversion of the mono-window algorithm. 


\subsection{Data preprocessing}

The GEE platform-based API programming screens the Landsat atmospheric apparent reflectance (TOA) image that had been subjected to radiometric calibration and atmospheric correction, called the SRTM DEM data in the GEE platform and calculated normalized vegetation index (NDVI) and Normalized Water Index (NDWI) were used as a characteristic variable for random forest classification to improve classification accuracy.

Visually interpreted high-resolution images of the three periods of 2010, 2014 and 2018 in Google Earth Pro, selected samples and classify land use types in Beijing-Tianjin-Hebei area into construction land, vegetation, and unused For the four categories of land and water, selected appropriate sample points according to the area ratio of various land use types in the study area (Table 1)

The sample points were exported from Google Earth Pro in kml format, converted to shp format in ArcMap 10.2, and imported into the GEE API for calling $70 \%$ of them as training samples and $30 \%$ as verification samples.

\begin{tabular}{ccc}
\hline Land use & Description & Number of samples \\
\hline Construction land & Urban land, rural settlements, roads & 20 \\
Vegetation & Forest, grassland, cultivated land & 25 \\
Unused land & Sandy land, bare land & 10 \\
Water body & River canals, lakes, reservoirs, tidal flats, wetlands & 15 \\
\hline
\end{tabular}

Table 1 Types of land use and number of different types of land use

\section{METHOD}

\subsection{GEE-based random forest land use classification}

The random forest algorithm is a flexible and easy-to-use machine learning algorithm. Even if parameter tuning is not performed, better classification accuracy can be obtained (Breiman, 2001). The basic principle is: (1) Using the Bootstrap method, the $\mathrm{N}$ sets of training sets are extracted from the original data, and each set of training sets extracted is about $2 / 3$ of the whole. (2) The $\mathrm{N}$ sets of training sets are used to construct the decision tree, and then the forest consisting of $\mathrm{N}$ decision trees is generated. In the growth process of each decision tree, $\mathrm{m}(\mathrm{m}<\mathrm{M})$ are selected from all $\mathrm{M}$ feature variables, and the Gini coefficient is adopted. The small principle selects the optimal feature variable to divide the internal nodes. (3) The prediction results of the $\mathrm{N}$ decision trees are combined to determine the new sample category by voting. Each sampling process has $1 / 3$ of the data not drawn, called extra-bag data, which can be used for internal error estimation and $\mathrm{OOB}$ error.

In the GEE, the random forest classifier is called, and the land use type is used as the target variable. The number of decision trees (ntree $=500$ ) is set by using the SRTM DEM data and NDVI and NDWI as the characteristic variables input during the training process, the default number of variables (mtry) is the square root of the total number of characteristic variables
(Ghosh, 2014), and then the land use classification of the main urban area of Guilin.

\subsection{GEE-based surface temperature inversion}

\subsubsection{Vegetation coverage and surface specific emissivity}

Firstly, the NDVI in the study area of the three periods was calculated in the GEE, and then the vegetation coverage PV in the study area was calculated according to the NDVI. Finally, the NDVI threshold method proposed by Sobrino (Sun et al, 2013; Lin et al, 2012) was used to calculate the surface specific emissivity $\mathcal{E}$ (Formula 1). Calculated as follows:

$$
\left\{\begin{array}{c}
\mathrm{NDVI}=(N I R-R) /(N I R+R) \\
P_{V}=\left[\left(\mathrm{NDVI}^{-\mathrm{NDVI}}{ }_{\mathrm{Soil}}\right) /\left(\mathrm{NDVI}_{\mathrm{Veg}}{ }^{\left.\left.-\mathrm{NDVI}_{\mathrm{Soil}}\right)\right]}\right.\right. \\
\varepsilon=0.004 P_{V}+0.986
\end{array}\right.
$$

In the formula, $\mathrm{NDVI}_{\text {Soil }}$ is the NDVI value of the bare soil or no vegetation coverage area, and $\mathrm{NDVI}_{\mathrm{Veg}}$ is the NDVI value of the pixel completely covered by the vegetation, that is, the NDVI value of the pure vegetation pixel. The empirical value is selected as $\mathrm{NDVI}_{\text {Soil }}=0.05$, and $\mathrm{NDVI}_{\mathrm{Veg}}$ is 0.70 (the maximum pixel value when less than 0.70 ). When a pixel NDVI value is greater than 0.70 , the PV value is 1 ; when the NDVI is less than 0.05 , the $\mathrm{PV}$ value is 0 .

\subsubsection{Surface temperature}


In this paper, the Mono-window algorithm improved by the traditional radiation equation is used to invert the Land Surface Temperature (LST) in the main city of Guilin (Wang et al, 2015; Zhou et al, 2010). The USGS points out There is calibration instability in the TIRS 11 band (Qin et al, 2001a), so the Landsat TM/ETM+ band 6 used in the original algorithm is improved to the 10th band of the Landsat 8 image, and the parameters $\mathrm{a}$ and $\mathrm{b}$ in the formula 2 are corrected. The calculation formula (Qin et al, 2001b) is as follows:

$$
T=\left[a(1-C-D)+(b(1-C-D)+C+D) T_{10}-D T_{a}\right] / C
$$

In the formula, T is the actual surface temperature in K. $a$ and $b$ are -62.735657 and 0.434036, respectively (Qin et al, 2001b). $C$ $=\varepsilon \tau, D=(1-\tau)[1+(1-\tau) \tau], \mathcal{E}$ is surface emissivity, $\tau$ is atmospheric transmittance, $T_{10}$ is the pixel brightness temperature of the thermal infrared band 10 of Landsat 8 , in $\mathrm{K}$. In winter (November to January), the atmospheric mean temperature $\left(T_{a}\right)$ of the location of the main urban area of Guilin (middle latitude) and the temperature $\left(T_{0}\right)$ near the ground (about $2 \mathrm{~m}$ ) have the following linear relationship (Qin et al, 2001b):

$$
\begin{gathered}
T_{a}=\left[19.2704+0.91118 T_{0}\right] \\
T_{10}=K_{2} / \ln \left(1+K_{1} / L_{\lambda}\right)
\end{gathered}
$$

Where, $T_{a}$ and $T_{0}$ units are all $\mathrm{K}, \mathrm{K} 1$ and $\mathrm{K} 2$ can be obtained from the header file of Landsat 8 data, $\mathrm{K}_{1}=774.89, \mathrm{~K}_{2}=1321.08$.

$L_{\lambda}$ is brighten for the pixel of band 10 .
In summary, the Mono-window algorithm for surface temperature inversion requires three parameters: average atmospheric temperature, atmospheric transmittance, and surface radiation rate. The average atmospheric temperature can be determined by Equation 4, the atmospheric transmittance can be calculated from the atmospheric water vapor content, while the Landsat 8 data can be found on the NASA website (http://atmcorr.gsfc.nasa.gov); The surface radiation rate can be obtained by formule 1 .

\section{RESULTS}

\subsection{Land use dynamic change characteristics}

In order to verify the accuracy of the classification results, the verification points are selected in the high-resolution image in Google Earth Pro (60 verification points are selected in each period, 15 in each category), and the visual interpretation of the verification point feature category is entered into the GEE. Carry out classification accuracy verification and spatial consistency analysis. The results show that the verification accuracy in 2010 is $83.3 \%$; the verification accuracy in 2014 is $81.6 \%$; the verification accuracy in 2018 is $88.3 \%$. The classification accuracy is higher than $80 \%$, which satisfies the requirements of this paper.
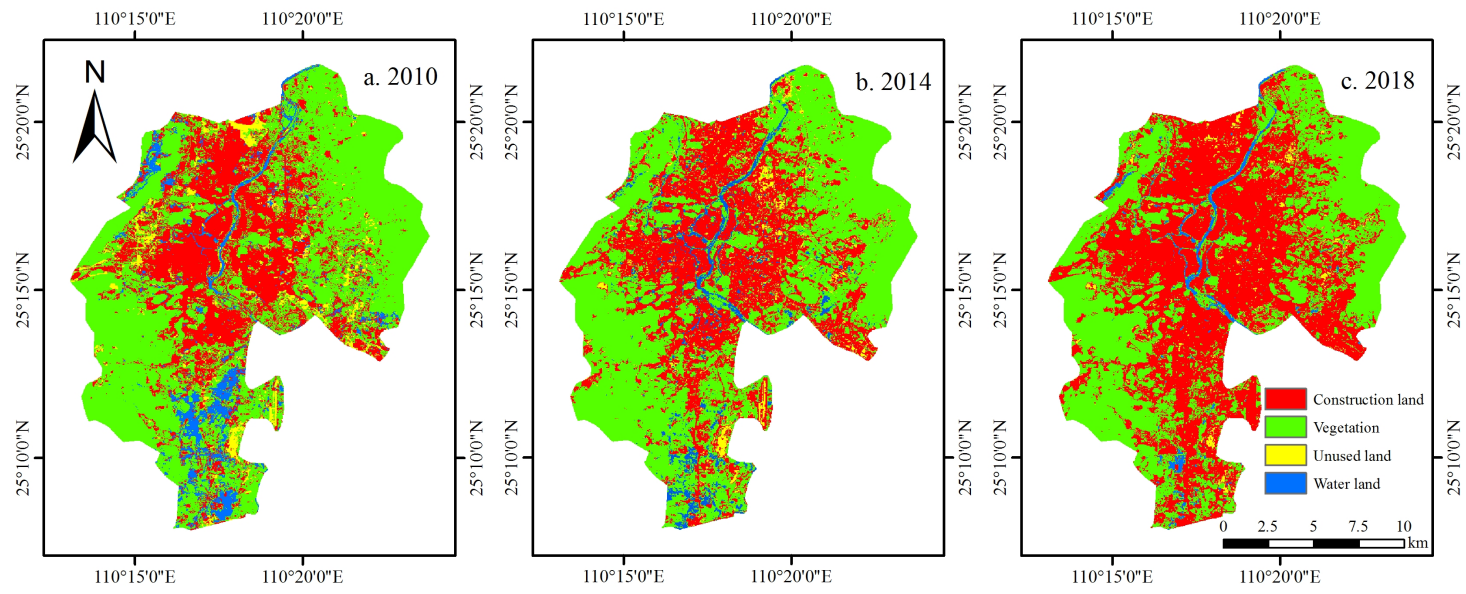

Figure 1 Land use type map of three periods in the main city of Guilin 

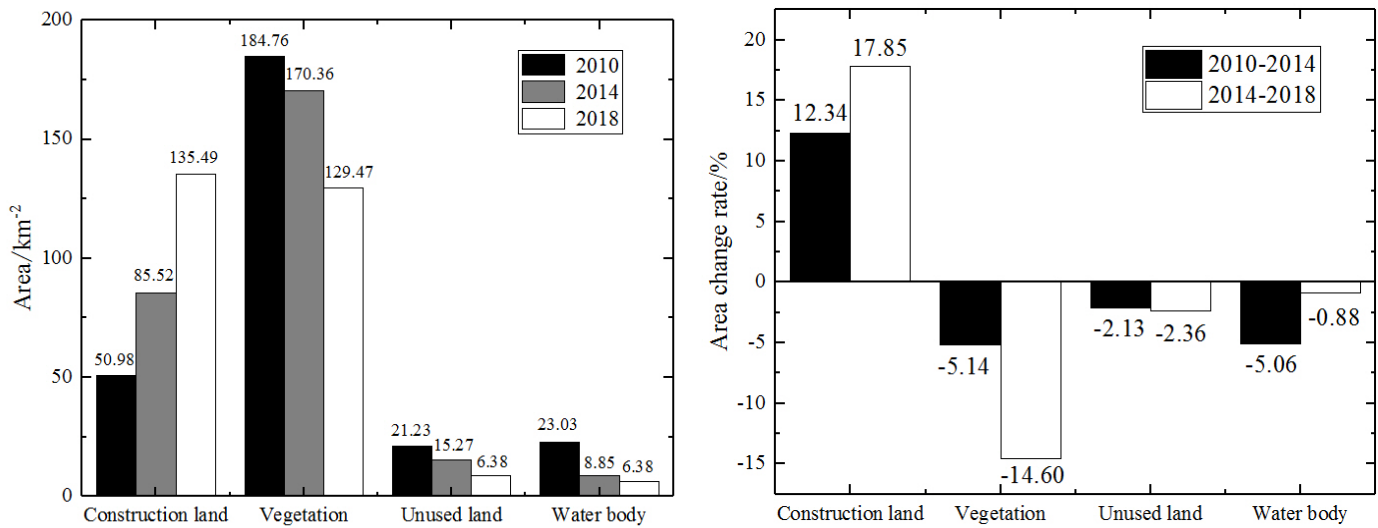

Figure 2 Land use type land area and area ratio change rate

It can be seen from Figure. 1 and Figure. 2 that the vegetation coverage in 2018 is reduced by $55.29 \mathrm{~km} 2$ compared with 2010 , the area ratio change rate is $-19.74 \%$, and the area is negative. The area of construction land in 2018 is $84.51 \mathrm{~km} 2$ compared with 2010. The rate of change is $30.19 \%$, and the area is growing. The area of unused land in 2018 is $14.85 \mathrm{~km} 2$ lower than that in 2010 . The rate of change in area is $-4.49 \%$, and the area is negative. The area of water in 2018 is $16.65 \mathrm{~km} 2$ less than 2010 . The rate of change was $-5.94 \%$, with a negative area growth. With the development of the city, human deforestation is used to build houses and roads (the construction land and unused land area grow rapidly), occupying rivers and ponds to make land around the lake (the water area is greatly reduced) and other activities seriously damage the main city of Guilin.
Ecological environment.

\subsection{Vegetation cover dynamic change characteristics}

In this paper, the vegetation cover in 2010, 2014 and 2018 is divided into five levels: high coverage, high coverage, medium coverage, low coverage and low coverage. It can be seen from the Figure.3 and Figure.4 that with the development of urbanization process, the area of high vegetation coverage has been greatly reduced, and the other four vegetation coverage grades have increased, and the vegetation coverage has continued to deteriorate.
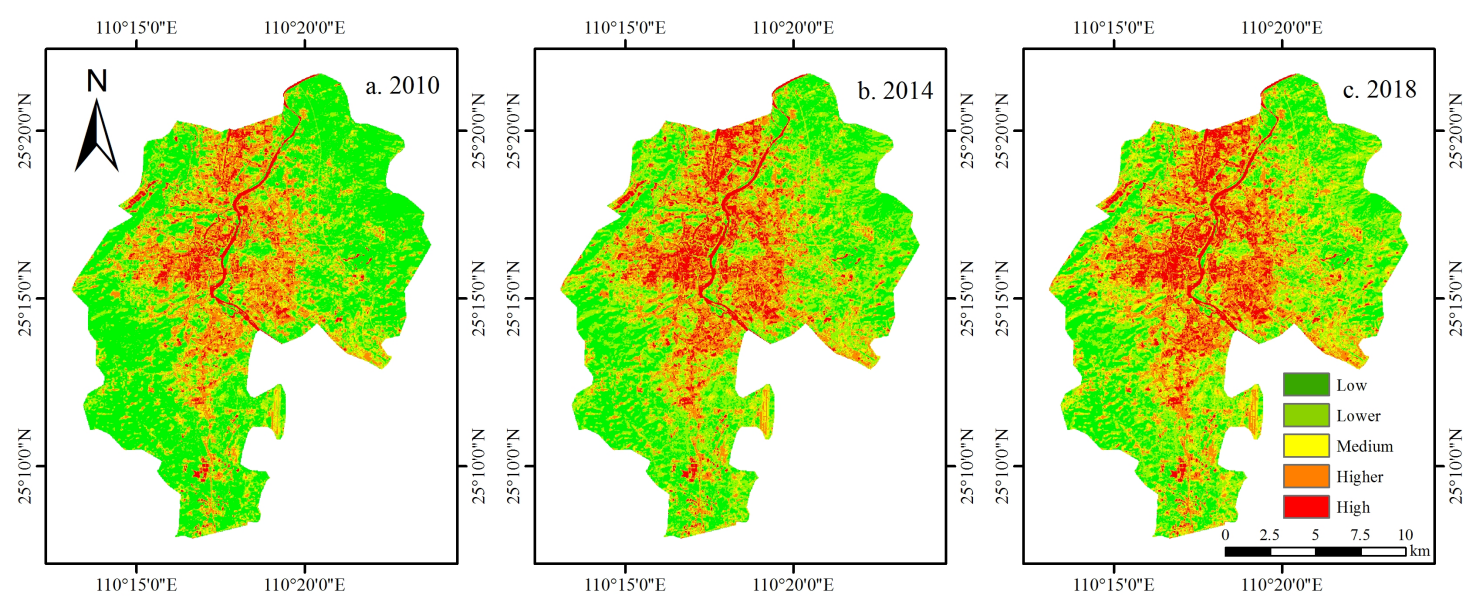

Figure 3 Vegetation coverage classification in three periods in the main urban area of Guilin 

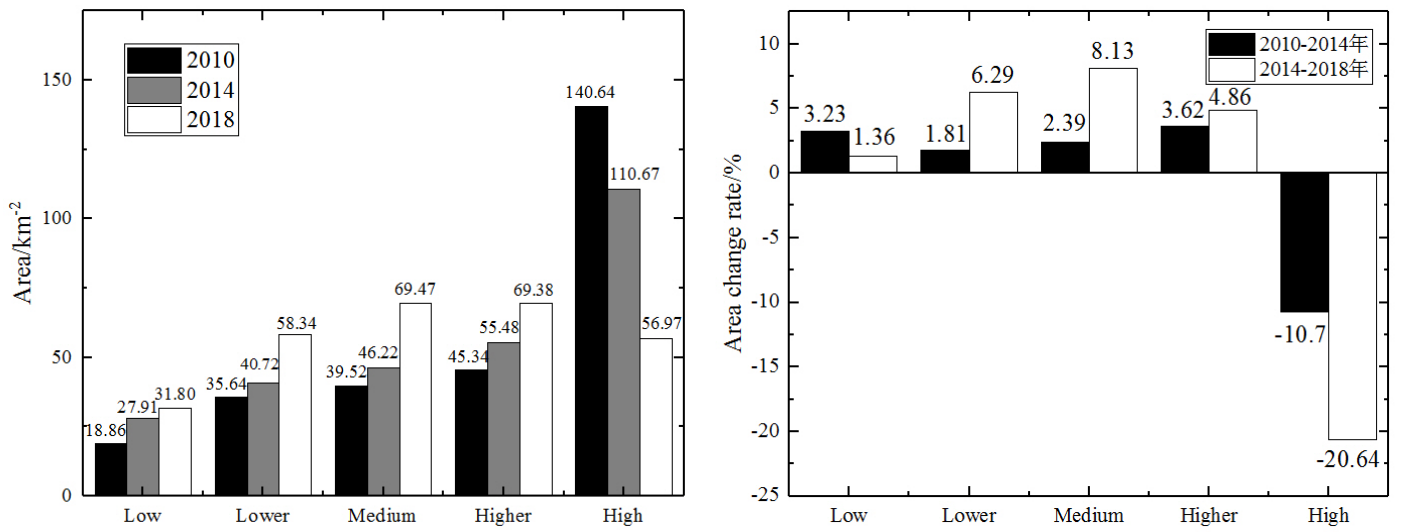

Figure 4 Vegetation coverage area and area ratio change rate at all levels

\subsection{Thermal environment dynamics}

In this paper, the surface temperatures in 2010, 2014 and 2018 are divided into five levels: high temperature zone, higher temperature zone, medium temperature zone, lower temperature zone and low temperature zone. It can be seen from the Figure.5 and Figure. 6 that with the development of urbanization process and the serious deterioration of vegetation coverage, the surface temperature of the main urban area of Guilin changes significantly, and the average temperature rises from $29.24{ }^{\circ} \mathrm{C}$ in 2010 and $29.67^{\circ} \mathrm{C}$ in 2014 . It is $30.53^{\circ} \mathrm{C}$ for 2018 .
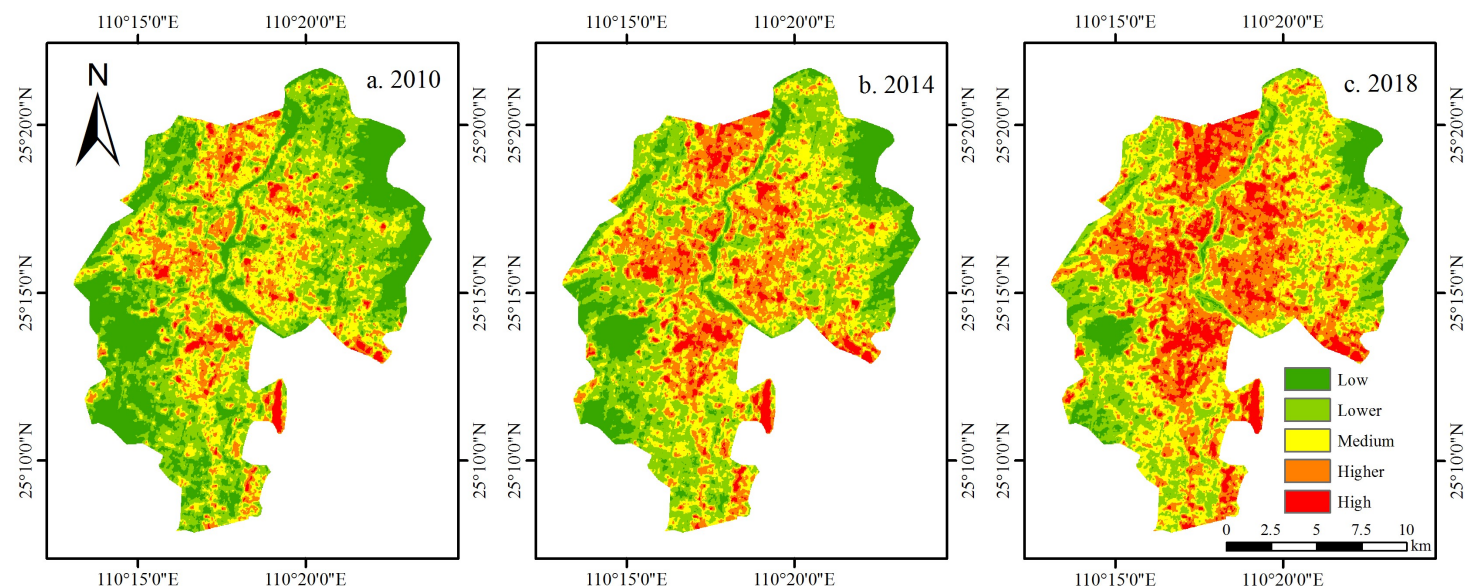

Figure 5 Surface temperature classification of three periods in the main city of Guilin
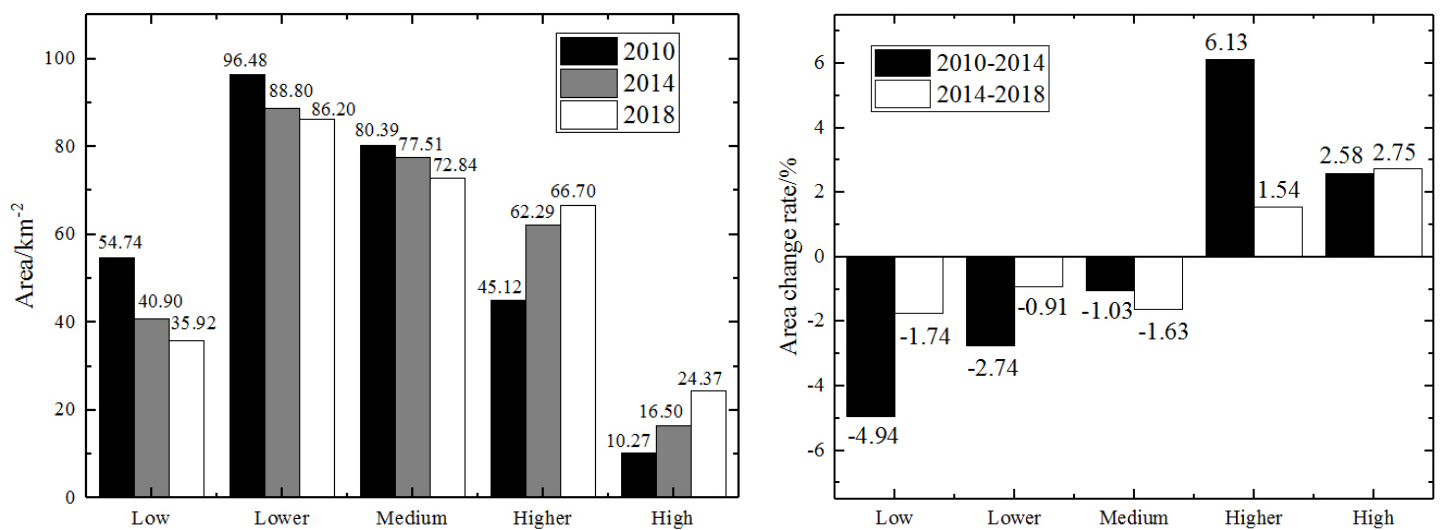

Figure 6 Area change and area ratio change rate of temperature zones at all levels 


\section{CONCLUSION}

In this paper, the Mono-window algorithm based on the GEE cloud platform is used to calculate the vegetation coverage and surface temperature of the main urban area of Guilin, and the results of the land use classification with random forest algorithm based on the GEE cloud platform are compared and analyzed.

(1) In 2010-2018, the average temperature of the main urban area of Guilin showed an upward trend, increasing by $1.29^{\circ} \mathrm{C}$. This is directly related to the decline in vegetation coverage caused by the urbanization process in the main urban area of Guilin.

(2) The lower temperature zone and the low temperature zone are mainly distributed in the vegetation and water body coverage areas, while the medium temperature zone, the higher temperature zone and the high temperature zone are mainly distributed in the construction land and the unused land cover area, so the vegetation coverage is increased, the water source is conserved and Reducing the unused land area can effectively alleviate the deterioration of the thermal environment in the main urban area of Guilin.

(3) In 2014-2018, the significant reduction of high vegetation coverage area is directly related to the further acceleration of urbanization. The area of high vegetation coverage decreased by $31.34 \%$, and the area of construction land increased by $30.19 \%$. The reduction of $4.49 \%$ of unused land area in the main urban area further illustrates the acceleration of urbanization construction. Land should be used rationally to reduce land waste and increase vegetation coverage through afforestation.

(4) The random forest algorithm based on GEE cloud platform not only has a fast calculation and has better classification accuracy. The verification accuracy in 2010, 2014 and 2018 is higher than $80 \%$, which is an effective land use classification. technology.

In summary, reduce the unused land area, increase vegetation coverage through afforestation, and thus affect Surface runoff to conserve water sources can effectively improve the gradual deterioration of the thermal environment in Guilin.

\section{ACKNOWLEDGEMENTS}

This study was funded by the National Natural Science
Foundation of China (Grant No. 41801071).

\section{REFERENCES}

Breiman L, 2001. Random forest. Machine Learning, 45(1):532.

Fu Y, Guo Q Z, Wu X X, 2018. Land surface temperature inversion in Tianjin city based on Landsat 8 remote sensing data. Environmental Monitoring in China, 34(5): 165-172.

Jimenez-Munoz J C, Sobrino J A, Skokovic D, Matter C, Cristobal J, 2014. Land surface temperature retrieval methods from Landsat-8 thermal infrared sensor data. IEEE Geoscience and Remote Sensing Letters, 11(10):1840-1843.

Hou G L, Zhang H Y, Wang Y Q, Qiao Z H, Zhang Z X, 2010. Retrieval and spatial distribution of land surface temperature in the middle part of Jilin province based on Modis data. Scientia Geographica Sinica, 30(3): 421-427.

Hou H R, Ding F, Li Q S. Remote sensing analysis of changes of urban thermal environment of Fuzhou city in China in the past 20 years. Journal of Geo-Information Science, 20(3): 385-395.

Hu D Y, Qiao K, Wang X L, Zhao L M, Ji G H, 2017. Comparison of three Single-window algorithms for retrieving land-surface temperature with Landsat 8 TIRS data. Geomatics and Information Science of Wuhan University, 42(7): 869-876.

Ghosh A, Sharma R, Joshi P K, 2014. Random forest classification of urban landscape using Landsat archive and ancillary data: Combining seasonal maps with decision level fusion. Applied Geography, 48(10): 31-41.

Liang B P, Li Y, Chen K Z, 2012. A research on land features and correlation between NDVI and land surface temperature in Guilin city. Remote Sensing Technology and Application, 27(3): 429-435.

Liu F, Wang X S, Xu J, Gao S J, 2012. Sensitivity analysis of retrieving land surface emissivity based on NDVI threshold method. Remote Sensing Information, 27(4): 3-12.

Maimaitijiang M, Alimujiang K, 2018. Spatial-temporal change of Urumqi urban land use and land cover based on grid cell approach. Transactions of the Chinese Society of Agricultural Engineering, 34(1): 210-216.

Meng X C, Li H, Du Y M, Cao B, Liu Q H, Li B, 2018. Retrieval and validation of the land surface temperature derived from Landsat 8 data: A case study of the Heihe River Basin. 
Journal of remote sensing, 22(5): 857-871.

Qin Z H, Karnieli A, Berliner P, 2001. A Mono-window algorithm for retrieving land surface temperature from Landsat TM data and its application to the Israel-Egypt border region[J]. International Journal of Remote Sensing, 22(18): 3719-3746.

Qin Z H, Zhang M H, Arnon Karnieli, Berliner P, 2001. Mono-window algorithm for retrieving land surface temperature from Landsat TM6 data. Acta Geographica Sinica, (4): 456-466.

Sun Z W, Tang B H, Wu Y, Cheng Y D, 2013. Retrieval and validation of land surface temperature with general Split-window algorithm from NOAA-18 (N) AVHRR/3 data. Journal of Geo-Information Science, 15(3): 431-439.

Tian H, Meng M, Wu M, Niu Z, 2019. Mapping spring canola and spring wheat using Radarsat- 2 and Landsat- 8 images with Google Earth Engine. Current science, 116(2):291-298.

Wang F, Qin Z, Song C, Tu L L, Karnieli A, Zhao S H, 2015. An improved Mono-window algorithm for land surface temperature retrieval from Landsat 8 thermal infrared sensor data. Remote Sensing, 7(4): 4268-4289.

Wang X X, Qin L M, Nong J H, Liang Z J, Zhu Q J, 2010. Land surface temperature retrieval with Mono-window algorithm in karst city. Journal of Guangxi Normal University (Natural Science Edition), 28(3): 10-14.

Weng Q P, Zhang H, Bao S X, Liu J G, Wu H, 2015. Study on ventilation channels of Nanjing city. Science Technology and Engineering, 15(11): 89-94.

Xie Q J, Chen K L, Jin G, 2017. Quantifying urbanization and its impact on urban heat island effect in Wuhan. Science of Surveying and Mapping, 42(9): 71-76+87.

Xiu X M, Zhou S F, Chen Q, Meng J H, Dong W Q, Yang G B, Li X S, 2019. Above-ground biomass estimation of provincial scattered grassland based on Google Earth Engine and machine learning. Bulletin of Surveying and Mapping, 0(3): 46-52+75.

$\mathrm{Xu} \mathrm{H} \mathrm{Q,} \mathrm{2015.} \mathrm{Retrieval} \mathrm{of} \mathrm{the} \mathrm{reflectance} \mathrm{and} \mathrm{land} \mathrm{surface}$ temperature of the newly launched Landsat 8 satellite. Chinese J. Geophys. 58(3): 741-747.

$\mathrm{Yu} \mathrm{X}$, Guo X, Wu Z. Land surface temperature retrieval from Landsat 8 TIRS - comparison between radiative transfer equation-based method, Split Window algorithm and Single Channel method. Remote Sensing, 6(10):9829-9852.

Zhang Z Z, Xiong K N, Huang D, 2018. Analysis on the spatiotemporal change and influence factors of vegetation cover in Fanjingshan Mountain over the last 30 years. Research of Soil and Water Conservation, 25(2): 183-189+389.
Zhou J, Zhan W F, Hu D Y, Zhao X, 2010. Improvement of Mono-window algorithm for retrieving land surface temperature from HJ-1B satellite data. Chinese Geographical Science, 20(2): 123-131. 